\title{
Oat Lines with Effective Adult Plant Resistance to Crown Rust
}

\author{
K. J. Leonard, U.S. Department of Agriculture, Agricultural Research Service, Cereal Disease Laboratory, Univer- \\ sity of Minnesota, St. Paul 55108
}

\begin{abstract}
Leonard, K. J. 2002. Oat lines with effective adult plant resistance to crown rust. Plant Dis. 86:593-598.

Race-specific resistance in oat does not provide adequate protection against crown rust because new virulent races of the pathogen often arise within a few years after cultivars with racespecific resistance are released. This study assessed levels of partial resistance in a series of experimental oat lines that showed reduced crown rust severity over 30 years of exposure to a diverse crown rust population. Oat breeding lines with putative adult plant slow-rusting resistance to Puccinia coronata were evaluated in small isolated field plots for crown rust severity in natural epidemics and in controlled inoculations in the greenhouse for infection efficiency with a single virulent $P$. coronata isolate. Lines were also tested as seedlings against isolates from a local $P$. coronata population to detect race-specific resistance. In field tests, areas under the disease progress curves for all 14 experimental lines tested were significantly less than for cv. Starter, the susceptible check, and values for nine of the lines were significantly less than for Portage, a standard slow-rusting cultivar. Four lines with good field resistance were susceptible as seedlings to $>85 \%$ of the local $P$. coronata population. One of the lines, MN841801, remained relatively free of rust in field plots over 7 years of tests, even in the 3 years when Starter was destroyed by crown rust. In greenhouse inoculations, adult plants of MN841801 developed only 7\% as many uredinia as Starter. MN841801 can be an excellent source of effective, durable adult plant resistance to crown rust.
\end{abstract}

Additional keywords: partial resistance

Crown rust, caused by Puccinia coro$n a t a$, is one of the most destructive diseases of oat in the United States. Over the past 10 years, yield losses in oat due to crown rust in the upper Midwest have generally been in the range of 1 to $5 \%$, but statewide, losses of 10 to $20 \%$ were reported for Iowa, Minnesota, and South Dakota from 1991 to 1993 (9). Losses of 10 to $20 \%$ were also reported for Louisiana in 1995 and 1997. Although an abundance of genes for race-specific resistance to crown rust are available in cultivated oat, Avena sativa, and the closely related wild relative, A. sterilis, the effectiveness of race-specific resistance has been shortlived in the United States and many other parts of the world (5). New pathogen races have overcome most new genes for racespecific resistance within a few years of their use in commercial cultivars. One reason for the short useful life span of

Corresponding author: K. J. Leonard E-mail: kurtl@cdl.umn.edu

Current address: Department of Plant Pathology, University of Minnesota, St. Paul 55108.

Accepted for publication 18 January 2002.

Publication no. D-2002-0322-01R

This article is in the public domain and not copyrightable. It may be freely reprinted with customary crediting of the source. The American Phytopathological Society, 2002 resistance genes is the widespread and common occurrence of Rhamnus cathartica, the aecial host for the sexual stage of $P$. coronata, throughout the Midwest, which serves not only as a source of primary inoculum, but also as the origin of new pathogenic races through genetic recombination.

The need for more durable resistance to crown rust in modern oat cultivars is clear. Partial resistance, often controlled by at least a few minor genes, is generally expected to be more durable than monogenic race-specific resistance characterized by hypersensitive reaction types (13). The prospect of using partial resistance of complex inheritance, however, is not always regarded as an appealing alternative to the use of simply inherited, hypersensitive resistance in breeding new oat cultivars. It takes more skill and effort to recognize and transfer partial resistance than hypersensitive resistance to new cultivars, particularly when the partial resistance is due to several genes with additive effects. Furthermore, the value of partial resistance is easily underrated in breeders' nurseries when compared with new sources of hypersensitive resistance. Typically, in screening nurseries there is an abundance of susceptible lines that provide sufficient inoculum to sustain a rust epidemic even on partially resistant lines.

A program was initiated at the University of Minnesota in the 1960s to enhance crown rust resistance in oat by combining different types of resistance from a variety of sources. The approach was described by Stuthman and Moore (20) as follows: "A series of crosses involving selected plants from the presumed cross Black Mesdag $\times$ S.P. 101 (obtained from F. A. Coffman) were made in 1959 and 1960. These selected plants were crossed to several lines from the Minnesota breeding program... The progeny were grown in the buckthorn nursery and selections were made using a pedigree system. Several different types of reactions as well as different levels of resistance were isolated. Presumably, most of the resistance was donated by the B.M. $\times$ S.P. 101 plants... The second phase was initiated in 1965 by choosing selections for a crossing program. Two lines which give an HR reading, 65B252-6 and 65B1362, one line with an MR-MS reaction, 65B2363, one line, 65B1286, characterized as a slow ruster (few small pustules which were slow to develop) and gave an MR reading and a line 65B1313, which formed teliospores very quickly (early telia) were selected. [Another line] of different origin [was] also included: a selection from Flo$\mathrm{rad} / 58-7$ (originally obtained from the Coker Pedigreed Seed Company) with an HR reaction, 65B663."

Because the oat lines from the Minnesota program combined multiple types of resistance, presumably including some genes for race-specific resistance, it was not clear how durable the resistance might be. The objectives of this study were to reexamine selected lines from the Minnesota program after 30 years of exposure to a diverse population of $P$. coronata in the St. Paul, MN buckthorn nursery and to assess their levels of partial and/or racespecific resistance to the current population of $P$. coronata.

\section{MATERIALS AND METHODS}

Oat lines. Fourteen oat lines were selected based on data from archived field books of M. B. Moore, University of Minnesota, and P. G. Rothman, USDA, ARS, Cereal Rust Laboratory, St. Paul, MN (Table 1). The 14 lines were tested in isolated field plots for resistance to crown rust; six of the lines were also tested in the greenhouse. In field and greenhouse tests, a susceptible check, cv. Starter (PI 502922), and a moderately resistant cultivar, Portage (CI 7107), were included for comparison. Starter is a short, early maturing oat with high yield and good lodging resistance that was released as a cultivar by the Minnesota Agricultural Experiment Station in 1986 
and registered in 1990 (21). When released, Starter was reported to have some resistance to crown rust, but by the early 1990s it was rated highly susceptible in field tests in St. Paul, MN. Portage was developed in Wisconsin (17) and became a standard for moderate slow-rusting resistance, which has retained its effectiveness since the 1960s $(6,15,18)$.

Twelve of the 14 breeding lines used in this study were regularly tested in the buckthorn (Rhamnus cathartica) nursery in St. Paul, where they were planted between hedges of $R$. cathartica, the alternate host for $P$. coronata. In the buckthorn nursery, the lines were exposed to severe disease pressure from a very diverse population of $P$. coronata initiated from the aecial infections on $R$. cathartica each spring. Oat straw bearing telia of $P$. coronata was overwintered and placed on top of the hedges each spring to induce heavy aecial infection (10). Severity of oat crown rust on lines in the nursery was recorded at milk to early dough stage (usually midJuly). P. G. Rothman's field books from 1976 to 1983 include crown rust severity data from tests in which 12 of the oat lines were planted in a block within the buck- thorn nursery each year, and Portage was planted adjacent to the block as a slowrusting standard for comparison. For these years, crown rust severity on the 12 lines never exceeded and usually was much lower than on Portage (Table 2). These tests included each line as a single row and were not replicated so it is not possible to compare entries statistically within years. Over the 8-year period, however, the mean crown rust severity recorded on each of the 12 lines was significantly lower $(P<0.01$, one-tailed paired $t$ test) than the mean value on Portage. The relatively high severity readings for lines with moderate resistance are attributed to the extreme disease pressure on oat plants from emergence to maturity in the buckthorn nursery. Fully susceptible oat lines typically produce little or no grain yield in the nursery.

Field tests. Oat lines were tested in isolated field plots in 1993 through 1999. In each year, there were 12 entries in four replications in a randomized complete block design. In some years, there were entries that are not relevant to the purposes of this study, so they are omitted from the results. Lines described in this study were included in at least 2 years of field tests.
Four entries, Starter, Portage, MN841801, and MN841810, were included every year. The field study consisted of six-row plots, $3.0 \mathrm{~m} \times 1.8 \mathrm{~m}$ separated on each side by 9 $\mathrm{m}$ of spring wheat, which is immune to oat crown rust. The field plot layout followed that of Parlevliet and van Ommeren (14) to minimize confounding effects of interplot interference. A hedge of $R$. cathartica annually infected with $P$. coronata aecia, and 20 to 30 rows of Starter oat adjacent to the hedge served as the inoculum source for natural infection of the plots each year. The hedge and the Starter spreader rows were approximately $20 \mathrm{~m}$ from the nearest plots. Treatments were replicated in blocks arranged in increasing distance from the hedge. The location of this field test was approximately $1.6 \mathrm{~km}$ northeast of the main St. Paul buckthorn nursery, which also may have provided some inoculum to the slow-rusting plots in most years. Virulence tests conducted over several years showed that frequencies of virulence to known crown rust resistance genes were similar between aecial isolates collected from the buckthorn nursery compared with those from the $R$. cathartica hedge near the slow-rusting plots (data not shown).

Table 1. Pedigrees of oat lines tested for partial resistance to crown rust

\begin{tabular}{ll}
\hline Line & Pedigree \\
\hline MN841402 & Lodi/4/LMHJA/Clintland//Rodney/3/Black Mesdag/Aberdeen 101 [Lodi/65B252-6] ${ }^{\mathrm{y}}$ \\
MN841443 & Black Mesdag/Aberdeen 101//CI7467/3/Rodney/4/LMHJA/Clintland //Rodney/3/Black Mesdag/Aberdeen 101 [65B1313/65B252-6] \\
MN841801 & Florad/Coker 58-7/3/CI7558//Black Mesdag/Aberdeen 101 [65B663/65B1362] \\
MN841804 & CI7683//Black Mesdag/Aberdeen 101/3/Rodney/4/CI7558//Black Mesdag/Aberdeen 101 [65B1286/65B1362] \\
MN841810 & Black Mesdag/Aberdeen 101//CI7467/3/Rodney/4/Florad/Coker 58-7 [65B1313/65B663] \\
MN841812 & LMHJA/Clintland//Rodney/3/Black Mesdag/Aberdeen 101/4/CI7683//Black Mesdag/Aberdeen 101/3/Rodney [65B252-6/65B1286] \\
MN841821 & Tippecanoe/3/Mn II 54-13//Black Mesdag/Aberdeen 101/4/CI8368 [Tippecanoe/65B252-6//CI8368] \\
MN841823 & Lodi/4/LMHJA/Clintland//Rodney/3/Black Mesdag/Aberdeen 101/5/Lodi//Florad/Coker 587 [Lodi/65B252-6//Lodi/65B663] \\
MN841825 & Tippecanoe/3/CI7558//Black Mesdag/Aberdeen 101/4/Tippecanoe //Florad/Coker 58-7 [Tippecanoe/65B1362//Tippecanoe/65B663] \\
MN841827 & Tippecanoe/4/CI7683//Black Mesdag/Aberdeen 101/3/Rodney/5/(unknown cross) ${ }^{\mathrm{z}}$ [Tippecanoe/65B1286//Lodi/(unknown cross)] \\
MN841832 & Lodi/4/LMHJA/Clintland//Rodney/3/Black Mesdag/Aberdeen 101/5/Lodi/(unknown cross) ${ }^{\mathrm{z}}[$ Lodi/65B252-6//Lodi/(unknown cross) \\
MN841837 & Lodi/3/CI7558//Black Mesdag/Aberdeen 101/5/Lodi/4/CI7683//Black Mesdag/Aberdeen 101/3/Rodney [Lodi/65B1362//Lodi/65B1286] \\
MN841841 & Tippecanoe/4/LMHJA/Clintland//Rodney/3/Black Mesdag/Aberdeen 101/5/Tippecanoe//Florad/Coker 58-7 [Tippecanoe/65B252- \\
& 6//Tippecanoe/65B663] \\
MN841842 & CI8368/4/Tippecanoe/3/CI7558//Black Mesdag/Aberdeen 101 [CI8368//Tippecanoe/65B1362] \\
\hline y Brackets indicate abbreviated pedigree. \\
z In field books, the unknown cross is listed as Lodi/4/CI7683//Black Mesdag/Aberdeen101/3/Rodney, but a note in the 1968 field book indicates that plants se- \\
lected are too compact to have come from that cross.
\end{tabular}

Table 2. Visual estimates of percentage crown rust severity ${ }^{z}$ on oat lines selected for partial resistance in the St. Paul, MN buckthorn nursery at milk to early dough stages

\begin{tabular}{|c|c|c|c|c|c|c|c|c|c|}
\hline \multirow[b]{2}{*}{ Oat line } & \multicolumn{9}{|c|}{ Crown rust severity $(\%)$} \\
\hline & 1976 & 1977 & 1978 & 1979 & 1980 & 1981 & 1982 & 1983 & average \\
\hline Portage & 30 & 50 & 60 & 70 & 60 & 60 & 60 & 60 & 55.7 \\
\hline MN841801 & 2 & 10 & 10 & 10 & 20 & 20 & 20 & 0 & 10.3 \\
\hline MN841804 & 20 & 20 & 50 & 10 & 40 & 50 & 50 & 20 & 30.0 \\
\hline MN841810 & 5 & 5 & 40 & 20 & 50 & 30 & 30 & 30 & 25.7 \\
\hline MN841812 & 10 & 30 & 60 & 40 & 50 & 40 & 40 & 20 & 35.7 \\
\hline MN841821 & 20 & 10 & 20 & 30 & 40 & 30 & 30 & 40 & 27.1 \\
\hline MN841823 & 10 & 5 & 20 & 10 & 30 & 30 & 30 & 50 & 22.1 \\
\hline MN841825 & 0 & 10 & 50 & 30 & 40 & 30 & 30 & 40 & 28.6 \\
\hline MN841827 & $\ldots$ & $\ldots$ & 10 & 20 & 40 & 50 & 50 & 30 & 30.0 \\
\hline MN841832 & 10 & 20 & 50 & 20 & 40 & 40 & 40 & 40 & 31.4 \\
\hline MN841837 & 10 & 20 & 50 & 10 & 50 & 30 & 30 & 40 & 30.0 \\
\hline MN841841 & 10 & 2 & 50 & 5 & 40 & 20 & 20 & 40 & 23.9 \\
\hline MN841842 & 0 & 10 & 60 & 30 & 50 & 40 & 40 & 30 & 31.4 \\
\hline
\end{tabular}

${ }^{\mathrm{z}}$ Data are from field books of P. G. Rothman, formerly of the USDA ARS, Cereal Rust Laboratory, St. Paul, MN. 
Field plots were planted in May each year, the date depending on the weather and availability of field equipment. When crown rust pustules were observed in late June or early July, the number of uredinia on the top three leaves of five plants in each of three locations in each plot was counted. Counts were repeated two or three times per week until the flag leaves of Starter, the susceptible check, began to die. When crown rust severities exceeded several hundred pustules per plant, the percentage of severity was estimated by comparing infected leaves to diagrammatic scales of $1,5,10,20,30,40,50$, $60,70,80,90$, or $100 \%$ infection, based on $37 \%$ coverage of the total leaf surface with pustules at $100 \%$ infection. Each $1 \%$ severity of crown rust infection was considered equivalent to an average of 15 uredinia per leaf. Telia were excluded from the counts because they represented pustules no longer contributing to disease increase. For each entry, the area under the disease progress curve (AUDPC) was calculated as:

$$
\text { AUDPC }=0.5 \sum_{1}^{n}\left(t_{i+1}-t_{i}\right)\left(y_{i+1}+y_{i}\right)
$$

where the day of the $i$ th reading is indicated by $t_{i}$, with $i=1$ for the first reading, $i$ $=2$ for the second, etc., and where $y_{i}$ is the mean number of uredinia per plant per plot at the $i$ th reading. For purposes of graphical presentation, 20 June was recorded as day 1 in each year. Treatment means were compared statistically by Duncan's multiple range test (19). In addition, the mean AUDPC values for MN841801, MN841810, and Portage were compared over all replications for all 7 years from 1993 to 1999 by one-tailed, paired $t$ tests of the null hypotheses that the mean AUDPC value for Portage was not greater than MN841801 or MN841810 and that the mean AUDPC value for MN841810 was not greater than MN841801.

To determine whether the field resistance was due to adult plant resistance or to race-specific seedling resistance, all of the oat lines except MN841842 were tested in the greenhouse as seedlings for susceptibility to isolates of $P$. coronata collected from the St. Paul buckthorn nursery. These tests were done in conjunction with a virulence survey in which field and nursery isolates of $P$. coronata were tested on a standard set of 28 differential lines. For the buckthorn nursery isolates, the slowrusting lines were added to the differential set to determine the proportion of the $P$. coronata population in the St. Paul area virulent on each slow-rusting line. Lines MN841801, MN841804, MN841810, MN841812, MN841821, MN841823, and MN841837 were tested against 70 isolates from 1992, and lines MN841402, MN841443, MN841825, MN841827, MN841832, and MN841841 were tested against 39 isolates from 1997.
Greenhouse tests. Adult plants of Starter, Portage, MN841801, MN841804, MN841810, MN841812, and MN841823 were grown in the greenhouse in soil in 15$\mathrm{cm}$ diameter pots. Plants were thinned to three per pot. There were three pots of each line in each of four replicated trials planted on 12 March, 19 March, 29 March, and 5 April. When flag leaves were fully emerged, the plants were inoculated with a single-pustule isolate, 92MNB-181, which was virulent on all of the lines in the test when inoculated as seedlings. Isolate 92MNB-181 was derived by collecting spores from a single uredinium on a seedling of the susceptible cultivar Marvellous that had been inoculated in the greenhouse with aeciospores from a single aecium collected in 1992 from the St. Paul buckthorn nursery.

Pots with the adult test plants were randomized and then sprayed with a suspension of urediniospores in light mineral oil with several passes over the plants in an effort to distribute the inoculum evenly.
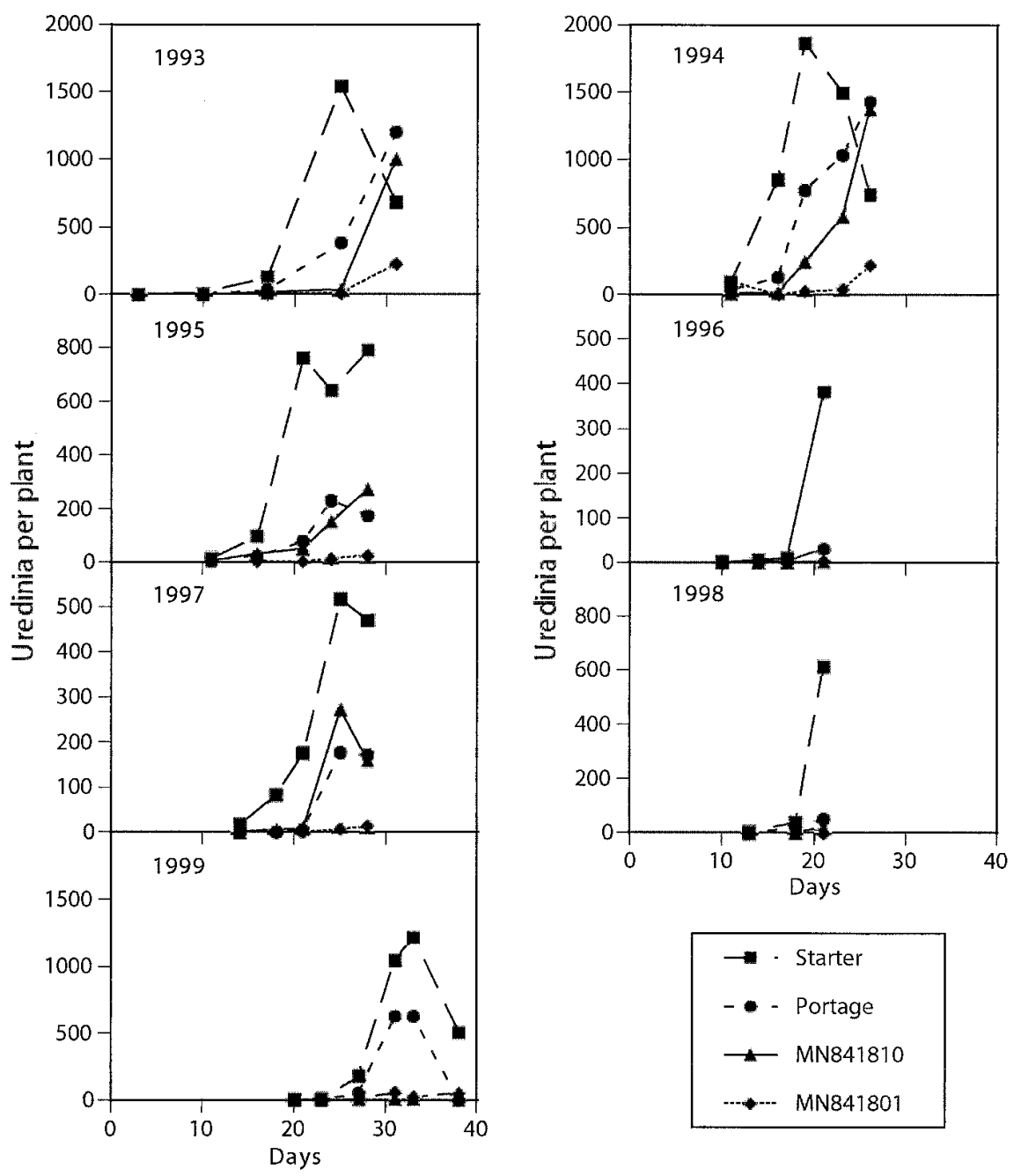

Fig. 1. Crown rust disease progress in two slow-rusting oat breeding lines (MN841801 and MN841810), a susceptible check (Starter), and a moderately resistant check (Portage) cultivar in natural epidemics in small isolated field plots over 7 years. Data are mean numbers of uredinia on the top three leaves per plant over four replications; 20 June is arbitrarily selected as day 1 . Note differences in scale from year to year.
After the oil had evaporated (approximately $1 \mathrm{~h}$ ), the plants were placed in a mist chamber overnight. In the morning the chamber was opened to allow the plants to dry gradually, and the plants were returned to the greenhouse. Inoculations for the four trials were done on 14 April, 21 April, 6 May, and 20 May.

The numbers of sporulating uredinia on each plant were counted at intervals of 2 to 3 days from 12 to 29 days after inoculation. After 19 or 20 days there was no further increase in the number of uredinia counted. Mean numbers of uredinia per plant of each entry on day 19 or day 20 were compared by Duncan's multiple range test for each trial. Correlations between tests in the greenhouse, as well as correlations between mean numbers of uredinia per plant for lines in the greenhouse and AUDPC in the field in 1993 and 1994, were calculated with Microsoft Excel.

To confirm the observation that there was little difference in latent period for 
crown rust on the lines, a fifth set of plants (planted 12 April) was inoculated on 6 June, and the date of first appearance of sporulation was recorded for each line. The first sporulating uredinium was observed 6 days after inoculation on Starter, MN841804, and MN841823; 7 days after inoculation on MN841812 and MN841821; and 8 days after inoculation on MN841801, MN841810, and Portage.

During the period of the experiments, temperatures in the greenhouse generally ranged between lows of $15^{\circ} \mathrm{C}$ at night to highs of $27^{\circ} \mathrm{C}$ in the afternoon. From 8 June to 13 June the daily high temperatures were in the range of 30 to $34^{\circ} \mathrm{C}$ and the low temperatures were in the range of 17 to $20^{\circ} \mathrm{C}$. The high temperatures shortly after inoculation of the fifth set of plants, no doubt, accounts for the early appearance of uredinia in that trial rather than the 8 to 10 days usually required on adult plants.

\section{RESULTS}

Field tests. Crown rust epidemics in the isolated plots were severe in 1993 and 1994 and moderately severe in 1995 and 1999 (Fig. 1). The epidemics in 1996 and 1998 were cut short when storms in midJuly caused the plots to lodge so badly that most plants did not recover. Little rust developed in the plots in 1997. For all seven epidemics, the numbers of uredinia per plant remained low on MN841801 throughout the season. MN841810 was less resistant than MN841801, but in most years the amount of rust on MN841810 was less than that on Portage, the moderately resistant check. In the severe epidemics of 1993 and 1994, however, crown rust increased rapidly on MN841810 at the end of the season, and at the time of the last

Table 3. Areas under the disease progress curves (based on uredinia per plant) for crown rust in isolated plots of oat lines selected for partial resistance in four years with natural infection

\begin{tabular}{lccccc}
\hline & \multicolumn{5}{c}{ Area under the disease progress curve } \\
Line & Isolates virulent $\mathbf{( \% )}$ & $\mathbf{1 9 9 3}$ & $\mathbf{1 9 9 4}$ & $\mathbf{1 9 9 5}$ & $\mathbf{1 9 9 6}$ \\
\hline Starter & 100 & $13,878 \mathrm{a}$ & $16,939 \mathrm{a}$ & $4,277 \mathrm{a}$ & $826 \mathrm{a}$ \\
Portage & $100^{*} \mathrm{y}$ & $6,530 \mathrm{~b}$ & $5,869 \mathrm{~b}$ & $793 \mathrm{~b}$ & $84 \mathrm{~b}$ \\
MN841443 & $90^{*}$ & $\ldots$ & $\ldots$ & $811 \mathrm{~b}$ & $14 \mathrm{~b}$ \\
MN841810 & $97^{*}$ & $3,347 \mathrm{c}$ & $2,287 \mathrm{c}$ & $604 \mathrm{~b}$ & $2 \mathrm{~b}$ \\
MN841823 & 33 & $2,468 \mathrm{~cd}$ & $1,957 \mathrm{~cd}$ & $\ldots$ & $27 \mathrm{~b}$ \\
MN841842 & $\ldots$ & $\ldots$ & $1,349 \mathrm{cde}$ & $72 \mathrm{~b}$ & $\ldots$ \\
MN841821 & 43 & $970 \mathrm{de}$ & $782 \mathrm{cde}$ & $\ldots$ & $\ldots$ \\
MN841402 & $97^{*}$ & $\ldots$ & $\ldots$ & $525 \mathrm{~b}$ & $42 \mathrm{~b}$ \\
MN841832 & 51 & $\ldots$ & $\ldots$ & $297 \mathrm{~b}$ & $3 \mathrm{~b}$ \\
MN841841 & 24 & $\ldots$ & $\ldots$ & $98 \mathrm{~b}$ & $5 \mathrm{~b}$ \\
MN841827 & 18 & $769 \mathrm{de}$ & $459 \mathrm{cde}$ & $72 \mathrm{~b}$ & $5 \mathrm{~b}$ \\
MN841801 & $94 *$ & $151 \mathrm{e}$ & $447 \mathrm{cde}$ & $\ldots$ & $7 \mathrm{~b}$ \\
MN841825 & 26 & $646 \mathrm{de}$ & $197 \mathrm{de}$ & $\ldots$ & $\ldots$ \\
MN841812 & 54 & $535 \mathrm{de}$ & $142 \mathrm{de}$ & $\ldots$ & $\ldots$ \\
MN841804 & $88^{*}$ & $\ldots$ & $17 \mathrm{e}$ & $28 \mathrm{~b}$ & $\ldots$ \\
MN841837 & 15 & & $\ldots$
\end{tabular}

${ }^{\text {w}}$ Means followed by the same letter are not significantly different according to Duncan's multiple range test $(P<0.05)$.

${ }^{x}$ Percentage of isolates of Puccinia coronata collected from the St. Paul, MN buckthorn nursery virulent on indicated oat lines in the seedling stage in greenhouse tests.

${ }^{y}$ Entries marked with $*$ are oat lines considered to have adult plant slow-rusting resistance; other lines may have a combination of race-specific resistance and nonspecific slow-rusting resistance.

${ }^{\mathrm{z}}$ Not tested. rust reading there was little difference in MN841810. The decline in numbers of sporulating uredinia on Starter late in the seasons in 1993, 1994, 1995, 1997, and 1999 was caused by conversion of uredinia death of severely rusted leaves. plant were not included in comparisons of AUDPC for any of the lines in those years.

AUDPC values of the oat lines tested in 1993 to 1996 were compared (Table 3). were not included because there was insufficient rust in consecutive years to permit comparisons over 2 years for those lines. Among the lines tested in 1993 to 1996 , MN841804, MN841810, MN841443, and Portage was considered to be predominantly, if not exclusively, slow-rusting, because they were susceptible to $88 \%$ or higher of the P. coronata isolates tested
from the St. Paul buckthorn nursery. In addition, MN841821, MN841832, and least 1812 were regarded as having at because they were susceptible to about one-half of the isolates.

All of the resistant lines had signifi-
cantly lower AUDPC values $(P<0.05)$ than Starter, the susceptible check, every year (Table 3). In 1995 and 1996, when AUDPC values were low or relatively low,
none of the resistant lines differed significantly $(P=0.05)$ from Portage, but in 1993 tested had significantly less rust than Portage. The lower AUDPC values for MN841810 were due to the delayed increase of rust relative to Portage (Fig. 1). to telia and in 1993, 1994, and 1999, by
Crown rust severities on MN841801, MN188404, MN841812, MN841825, and MN841837 did not increase substantially in any year. MN841837, which had significantly lower AUDPC than MN841810 in 1994, was not tested in 1993. The lower AUDPC value of MN841825 and MN841837 may have been due largely to race-specific resistance, because only approximately 26 and $15 \%$ of the isolates in the St. Paul area could overcome the racespecific resistance of MN841825 and MN841837.

Mean AUDPC values for MN841801, MN841810, and Portage over all 7 years from 1993 to 1999 were 215, 1026, and 1755, respectively. Mean AUDPC for Portage was significantly greater than that for MN841801 $(P=0.001)$ and MN841810 $(P \geq 0.040)$ as determined in one-tailed, paired $t$ tests over the 28 plots per line (4 replications $\times 7$ years). In addition, the mean AUDPC for MN841801 was significantly less than that for MN841810 ( $P=$ 0.003). AUDPC values for 1993 and 1994 were highly correlated for those lines tested in both years $\left(r^{2}=0.984\right)$. This was true even with the highly susceptible Starter removed from the analysis $\left(r^{2}=\right.$ 0.988). AUDPC values for 1995 and 1996 also were highly correlated for the lines tested in those years $\left(r^{2}=0.965\right)$. Much of the high correlation for 1995 and 1996, however, was due to inclusion of Starter, which had higher AUDPC values than the other lines. When Starter was removed from the analysis for 1995 and 1996, the correlation dropped to $r^{2}=0.336$. Thus, the relative AUDPC values of lines in 1996 were not indicative of the abilities of the lines to resist crown rust under conditions of moderate disease pressure as in 1995.

Greenhouse tests. The four greenhouse tests produced a wide range of mean numbers of uredinia per plant, from 6.4 for Starter in test 1 to 184.3 for Starter in test 3 (Table 4). In contrast to the field tests in which the levels of resistance of MN841810 and MN841823 appeared similar, MN841823 appeared significantly less resistant than MN841810 in three of four greenhouse tests. The difference between field and greenhouse tests may reflect the high proportion of field isolates that were avirulent on MN841823 (Table 3), whereas a single, virulent isolate was chosen for the greenhouse inoculations. In the greenhouse, the mean number of uredinia per plant on MN841823 was significantly greater than on Starter $(P<0.05)$ in greenhouse test 1, fewer than Starter in tests 3 and 4 , and did not differ significantly from Starter in test 2 (Table 4).

Several lines showed moderate to high levels of resistance in the greenhouse tests (Table 4). Over all four greenhouse tests, MN841801 had only $7.3 \%$ (range 2.1 to $18.2 \%$ ) as many uredinia as Starter; MN841810 had $12.9 \%$ (range 3.1 to 
$21.8 \%$ ) as many; and Portage had $15.1 \%$ (range 1.9 to $28.0 \%$ ) as many as Starter. In greenhouse tests, Portage appeared more resistant than in the field tests, even though essentially all $P$. coronata isolates in the field were virulent on Portage.

In the greenhouse, it was apparent that MN841804 is a mixture of types with high and low levels of adult plant resistance to crown rust. In tests 2 to 4 there were sufficient numbers of uredinia to clearly distinguish plants with high and low levels of resistance; $42 \%$ of the plants had large numbers of uredinia, $58 \%$ had few uredinia, and there were no intermediate types. Furthermore, the uredinia on plants with many uredinia were moderately large with abundant sporulation, whereas those on plants with few uredinia were small with only moderate sporulation. In spite of having $42 \%$ plants with little resistance, the mean number of uredinia per plant on MN841804 over all four greenhouse tests was only $28.5 \%$ (range 14.6 to $48.2 \%$ ) of that on Starter.

Although statistically significant in five of six comparisons, the correlations for mean numbers of uredinia on lines across tests in the greenhouse were not high, perhaps due to the large range in infection severity across tests. The $r^{2}$ values ranged from $0.666(P<0.01)$ between tests 2 and 4 , which had moderately low and moderately high rust severity, to $0.232(P>0.05)$ for tests 1 (lowest severity) and 3 (highest severity). The mean number of uredinia per plant in the greenhouse was poorly correlated with AUDPC values for the same lines in the field in 1993 and 1994 in three of the greenhouse tests, but the mean values over all four greenhouse tests were moderately correlated with field results (Table 5). One reason that the correlations were not higher was that MN841812, MN841821, and MN841823 all performed better in the field than expected from the results of the greenhouse tests. These lines were resistant to 46,57 , and $67 \%$, respectively, of $P$. coronata isolates collected from the St. Paul buckthorn nursery. When these three lines were removed from the comparisons, the correlation coefficients for mean values over all four greenhouse tests and the 1993 and 1994 field tests were $0.822(P<0.05)$ and $0.894(P<$ $0.01)$, respectively.

\section{DISCUSSION}

Observations from P. G. Rothman's field notes on single-row, unreplicated tests in the St. Paul buckthorn nursery from 1976 to 1983 suggested that 12 oat lines from the Minnesota oat breeding program had higher levels of resistance to crown rust than Portage, a standard for slow-rusting resistance among released cultivars. Replicated tests of these and two additional lines in isolated field plots confirmed that several of the lines are significantly more resistant than Portage. The partial resistance of the 12 oat lines appeared much more effective in the isolated plots than under conditions of intense interplot interference in the buckthorn nursery. For example, over 7 years of small plot tests, MN841801 remained relatively free of rust, even in years when crown rust completely destroyed the leaves of the susceptible check cultivar Starter. In 1993 and 1994, the years with most severe crown rust epidemics, MN841801, MN841804, and MN841810 had significantly lower AUDPC values than Portage. The resistance of MN841810 appeared to break down late in the season. In 1993, 1994, 1995, and 1997, late season crown rust severities on MN841810 were similar to those on Portage. Several other lines, MN841812, MN841821, MN841823, and MN841825, also had significantly lower AUDPC values than Portage, but the degree to which the lower AUDPC can be attributed to partial resistance is uncertain because their resistance included racespecific resistance effective against a substantial portion of the $P$. coronata population in the field plots.

In field tests in which host lines were exposed to natural inoculum consisting of a diverse mixture of pathogen races, it was important to account for possible presence of hypersensitive, race-specific resistance in addition to partial, adult plant resistance in the test lines. As Parlevliet (12) demonstrated in a simple model, host lines with different genes for race-specific resistance that are effective against different proportions of the races in a mixed pathogen population can give the appearance of continuous variation in disease severity expected from quantitative partial resistance. In the present study, several of the oat lines owed at least some of their reduced
AUDPC values in field tests to the presence of race-specific resistance. For example, MN841823 in field tests appeared to have a level of adult plant resistance equivalent to that of MN841810. When tested against individual isolates of $P$. coronata collected from the St. Paul buckthorn nursery, however, MN841823 in the seedling stage was resistant to $67 \%$ of the isolates, whereas, MN841810 was susceptible to $97 \%$ of the isolates. Furthermore, in inoculations with a single virulent isolate of $P$. coronata in the greenhouse, adult plants of MN841810 developed significantly fewer uredinia than those of MN841823 in three of four tests, and for all four tests, MN841810 averaged fewer than $40 \%$ of the number of uredinia on MN841823. The greenhouse tests support the conclusion that MN841823 probably has some adult plant resistance relative to the susceptible check Starter, but the level of resistance seems to be lower than expected from the 1976 to 1983 tests in the St. Paul buckthorn nursery (Table 2).

Of all the lines tested, the field plot performances of MN841443, MN841801, MN841402, and MN841810 are most representative of their true level of adult plant resistance. Based on the greenhouse tests, MN841804 is a mixture of genotypes, some of which have a high level of adult plant resistance that may be as good or better than that of MN841801. We have made single-plant selections from MN841804 plants showing high levels of adult plant resistance in greenhouse tests, and we are currently testing them in the field. On the basis of the epidemics in

Table 5. Correlation coefficients between crown rust severity on oat lines ${ }^{\mathrm{x}}$ in greenhouse tests and area under the disease progress curve in field tests in 1993 and 1994

\begin{tabular}{lll}
\hline & \multicolumn{2}{c}{ Field tests } \\
\cline { 2 - 3 } Greenhouse test & $\mathbf{1 9 9 3}$ & $\mathbf{1 9 9 4}$ \\
\hline 1 & 0.132 & 0.150 \\
2 & 0.212 & 0.290 \\
3 & $0.856^{* * \mathrm{y}}$ & $0.914^{* *}$ \\
4 & 0.182 & 0.246 \\
Greenhouse mean & $0.559^{* \mathrm{z}}$ & $0.644^{* *}$ \\
\hline
\end{tabular}

$\mathrm{x}$ Oat lines included in the tests are shown in Table 3 .

y ** = significantly greater than 0 at $P<0.01$

$\mathrm{z} *$ = significantly greater than 0 at $P<0.05$.

Table 4. Numbers of uredinia per plant ${ }^{\mathrm{z}}$ on adult oat plants inoculated in the greenhouse with a single virulent isolate of Puccinia coronata.

\begin{tabular}{|c|c|c|c|c|c|c|c|}
\hline \multicolumn{2}{|r|}{ Test 1} & \multicolumn{2}{|r|}{ Test 2} & \multicolumn{2}{|r|}{ Test 3} & \multicolumn{2}{|r|}{ Test 4} \\
\hline Oat line & Uredinia per plant & Oat line & Uredinia per plant & Oat line & Uredinia per plant & Oat line & Uredinia per plant \\
\hline MN841823 & $9.8 \mathrm{a}$ & Starter & $38.6 \mathrm{a}$ & Starter & $184.3 \mathrm{a}$ & Starter & $97.0 \mathrm{a}$ \\
\hline Starter & $6.4 \mathrm{~b}$ & MN841823 & $24.5 \mathrm{ab}$ & MN841821 & $54.3 \mathrm{~b}$ & MN841821 & $88.4 \mathrm{a}$ \\
\hline MN841821 & $2.9 \mathrm{bc}$ & MN841812 & $21.7 \mathrm{ab}$ & Portage & $51.6 \mathrm{~b}$ & MN841823 & $60.6 \mathrm{~b}$ \\
\hline MN841812 & $1.5 \mathrm{c}$ & MN841804 & $18.6 \mathrm{~b}$ & MN841823 & $48.4 \mathrm{bc}$ & MN841812 & $37.4 \mathrm{bc}$ \\
\hline MN841804 & $1.4 \mathrm{c}$ & MN841821 & $15.2 \mathrm{bc}$ & MN841810 & $40.2 \mathrm{bc}$ & MN841804 & $27.8 \mathrm{~cd}$ \\
\hline Portage & $1.4 \mathrm{c}$ & MN841801 & $1.3 \mathrm{c}$ & MN841812 & $36.5 \mathrm{bc}$ & MN841810 & $13.3 \mathrm{~cd}$ \\
\hline MN841801 & $1.2 \mathrm{c}$ & MN841810 & $1.2 \mathrm{c}$ & MN841804 & $27.0 \mathrm{bc}$ & Portage & $8.2 \mathrm{~d}$ \\
\hline MN841810 & $0.8 \mathrm{c}$ & Portage & $0.8 \mathrm{c}$ & MN841801 & $10.5 \mathrm{c}$ & MN841801 & $2.0 \mathrm{~d}$ \\
\hline
\end{tabular}

${ }^{\mathrm{z}}$ Means followed by the same letter in a column are not significantly different according to Duncan's multiple range test $(P<0.05)$. 
small isolated plots, the resistance of MN841801 and MN841804 appears to be sufficient to protect against crown rust without additional genes for race-specific resistance. Preliminary reports indicate that two major and several minor quantitative trait loci (QTLs) account for the adult plant resistance of MN841801 (3,4,16).

The partial resistance of MN841801 and MN841804, as measured in isolated field plots in this study, appears to be more effective than that identified by Briére et al. (2) in Quebec, where the oat lines with the best partial resistance to crown rust had AUDPC values approximately 22 and $17 \%$ of those of the most susceptible lines. In 1993, 1994, 1995, and 1996, MN841801 had AUDPC values only 6, 3, 1, and $0.8 \%$ as great as that of Starter. It is possible, however, that interplot interference may have masked some of the effectiveness of resistance of lines in the study by Briére et al. (2).

MN841812 had low AUDPC values in the heavy crown rust epidemics of 1993 and 1994 (Table 3), and may have worthwhile adult plant resistance in addition to the race-specific resistance expressed against $54 \%$ of $P$. coronata isolates in the seedling stage. In three of four greenhouse tests, MN841812 developed significantly fewer uredinia than the susceptible check Starter.

This study was not intended to account for all possible components of partial resistance in the lines from the Minnesota breeding program. Brake and Irwin (1) found that uredinium density per leaf was the component that best differentiated between levels of crown rust resistance in oat cultivars in their tests. Cultivar Panfive with partial resistance showed little or no difference in latent period compared to susceptible cultivars in their test. Panfive had smaller uredinia with reduced sporulation, as well as fewer uredinia, compared to susceptible cultivars. As summarized by Parlevliet (11), these components of partial resistance are usually associated fairly strongly in rust resistance. Although the uredinia were not measured in the greenhouse tests of the current study, the uredinia on lines with lower numbers of uredinia per plant definitely appeared smaller than those on Starter. On MN841801 many of the infections produced only flecks and most uredinia that did develop remained small, whereas those on Starter continued to enlarge over the infectious period. The low infection efficiency in MN841801 likely contributes more to its field resistance than its latent period, which was preliminarily estimated at approximately 2 days longer than that of Starter. In Leonard and Mundt's model (8), an $80 \%$ reduction in numbers of uredinia would have an effect on the rate of crown rust increase equivalent to an 8 to 10 day increase in latent period.

The oat lines with partial resistance to crown rust evaluated in this study have poor agronomic characteristics. Generally, they are tall, late maturing, and poor yielding, which accounts for the fact that they have not been used much in breeding commercial cultivars. The challenge will be to transfer sufficient resistance from one or more of the lines without introducing the undesirable traits into acceptable oat lines. Current efforts with molecular markers to map the genes responsible for the resistance of MN841801 may make this possible. If this can be accomplished, it seems likely that the transferred resistance will prove to be durable based on the history of the lines analyzed in this study. They remained resistant over 35 years, while other lines gradually succumbed to crown rust in the St. Paul buckthorn nursery. Still, as Johnson (7) eloquently pointed out, resistance can be declared to be truly durable only after it has remained effective for a long time with widespread use in a large area with conditions favorable for disease development.

\section{ACKNOWLEDGMENT}

The superb technical assistance of Gerald Ochocki throughout this study is gratefully acknowledged.

\section{LITERATURE CITED}

1. Brake, V. M., and Irwin, J. A. G. 1992. Partial resistance of oats to $P$. coronata f. sp. avenae. Aust. J. Agric. Res. 43:1217-1227.

2. Briére, S. C., Kushalappa, A. C., and Mather, D. E. 1994. Screening for partial resistance to an isolate of crown rust (Puccinia coronata $\mathrm{f}$. sp. avenae) race 264 in oat cultivars and breeding lines. Can. J. Plant Pathol. 16:49-55.

3. Chong, J. 2000. Inheritance of resistance to two Puccinia coronata isolates in a partial resistant oat line MN841801. Acta Phytopathol. Entomol. Hung. 35:37-40.

4. Chnog, J. 2001. Genetics of resistance to crown rust in a partially resistant oat line MN841801. (Abstr.) Can. J. Plant Pathol.
23:192.

5. Chong, J., and Kolmer, J. A. 1993. Virulence dynamics and phenotypic diversity of $P u c$ cinia coronata f. sp. avenae in Canada from 1974 to 1990. Can. J. Bot. 71:248-255.

6. Heagle, A. S., and Moore, M. B. 1970. Some effects of moderate adult resistance to crown rust of oats. Phytopathology 60:461-466.

7. Johnson, R. 1984. A critical analysis of durable resistance. Annu. Rev. Phytopathol. 22:309-330.

8. Leonard, K. J., and Mundt, C. C. 1984. Methods for estimating epidemiological effects of quantitative resistance to plant diseases. Theor. Appl. Genet. 67:219-230.

9. Long, D. L., and Hughes, M. E. 2001. Small Grain Losses Due to Rust. USDA-ARS Cerea Dis. Lab., University of Minnesota, St. Paul. On-line publication CDL-EP\#007.

10. Moore, M. B. 1966. Buckthorns and oat breeding for resistance to crown rust. (Abstr.) Phytopathology 56:891.

11. Parlevliet, J. E. 1979. Components of resistance that reduce the rate of epidemic development. Annu. Rev. Phytopathol. 17:203-222.

12. Parlevliet, J. E. 1983. Can horizontal resistance be recognized in the presence of vertical resistance in plants exposed to a mixture of pathogen races? Phytopathology 73:379.

13. Parlievliet, J E. 1988. Strategies for the utilization of partial resistance for the control of cereal rusts. Pages 48-62 in Breeding Strategies for Resistance to the Rusts of Wheat, $\mathrm{N}$. W. Simmonds and S. Rajaram, eds. CIMMYT, Mexico D.F.

14. Parlievliet, J. E., and van Ommeren, A. 1975. Partial resistance of barley to leaf rust, $P u c$ cinia hordei. II. relationship between field trials, micro-plot tests and latent period. Euphytica 24:293-303.

15. Politowski, K., and Browning, J. A. 1978. Tolerance and resistance to plant disease: an epidemiological study. Phytopathology 68:1177-1185.

16. Portyanko, V. A, Chen, G., Rines, H. W. Phillips, R. L., Leonard, K. J., Ochocki, G E., and Stuthman, D. D. 2001. Genetic basis of partial resistance to crown rust in cultivated oat 'MN841801-1'. (Abstr.) Plant and Animal Genome IX Conf. On-line publication $\mathrm{P} 438$.

17. Shands, H. L., Arawinko, Z. M., and Forsberg, R. A. 1966. Registration of Portage oats. Crop Sci 6:388

18. Singleton, L. L., Moore, M. B., Wilcoxson, R. D., and Kernkamp, M. F. 1982. Evaluation of oat crown rust disease parameters and yield in moderately resistant cultivars. Phytopathology 72:538-540.

19. Steel, R. G. D., and Torrie, J. H. 1960. Principles and Procedures of Statistics. McGrawHill, New York.

20. Stuthman, D. D., and Moore, M. B. 1970. A unique approach to crown rust resistance. Oat Newsl. 21:23-24.

21. Stuthman, D. D., Wilcoxson, R. D., and Rines, H. W. 1990. Registration of Starter oat. Crop Sci. 30:1365. 